\title{
Transatlantica
}

Revue d'études américaines. American Studies Journal

\section{Voix du Sud : étude de trois autobiographies de femmes esclaves}

Dominique Aurélia

\section{(2) OpenEdition}

1 Journals

\section{Édition électronique}

URL : https://journals.openedition.org/transatlantica/6229

DOI : $10.4000 /$ transatlantica.6229

ISSN : 1765-2766

Éditeur

Association française d'Etudes Américaines (AFEA)

Référence électronique

Dominique Aurélia, "Voix du Sud : étude de trois autobiographies de femmes esclaves », Transatlantica [En ligne], 2 | 2012, mis en ligne le 23 mai 2013, consulté le 06 avril 2023. URL : http:// journals.openedition.org/transatlantica/6229; DOI : https://doi.org/10.4000/transatlantica.6229

Ce document a été généré automatiquement le 6 avril 2023.

\section{(c) (i) (9)}

Creative Commons - Attribution - Pas d'Utilisation Commerciale - Pas de Modification 4.0 International - CC BY-NC-ND 4.0

https://creativecommons.org/licenses/by-nc-nd/4.0/ 


\title{
Voix du Sud : étude de trois autobiographies de femmes esclaves
}

\author{
Dominique Aurélia
}

1 Les récits d'esclaves apparaissent aux États-Unis dès 1760 à la faveur des mouvements abolitionnistes anglais et américains, comme témoignages, dans une première période ${ }^{1}$, de la perte de liberté physique et, dans une deuxième période (première moitié du XIX siècle), comme dénonciation de l'horreur du système esclavagiste. Henry Louis Gates ${ }^{2}$ estime que plus d'une centaine de récits d'esclaves furent publiés avant la fin de la guerre de Sécession. Ces récits se révèlent être parfois des réécritures formelles des témoignages des esclaves recueillis lors des conférences organisées par des sociétés anti-esclavagistes. Ces textes servent à amplifier la voix de l'esclave, la transformant en instrument de propagande anti-esclavagiste pouvant modifier l'opinion publique dans certaines régions et développer outre-Atlantique, le soutien à la cause ${ }^{3}$ (Sekora, 1987, 501). C'est ainsi que la voix du subalterne sera retranscrite et que la parole de l'esclave s'ajoutera à celle des sans-voix. Comme le note Arlette Frund, l'acquisition d'une voix rend visible à la fois le maitre et l'esclave, matérialise l'immatérialité et l'inhumanité de l'esclavage, et déplace le discours sur la place publique (Frund, 2007). Dans les récits publiés au cours du XVIII ${ }^{\mathrm{e}}$ siècle (The Interesting Narrative of the Life of Olaudah Equiano, or Gustava Vassa, the African, 1789 ; Poems on Various Subjects, Religious, and Moral, 17734), l'ancien esclave aborde les contradictions morales et religieuses de l'esclavage, participant d'un travail de résistance à travers la dénonciation des théories, idéologies et discours sur la race exprimés au $x{ }^{\mathrm{e}}{ }^{\mathrm{e}}$ siècle. Lorsqu'il devient évident après l'abolition de la traite transatlantique en 1807, que le système ne s'effondrera pas de lui-même, un nouveau type de récit s'impose : celui des fugitifs ${ }^{5}$. En 1831, la diffusion $\mathrm{du}$ périodique The Liberator par le pro-abolitionniste William Lloyd Garrison, va contribuer à la publication d'un certain nombre de récits de fugitifs. Par le biais de ces récits, les fugitifs attaquent directement l'institution dont ils étaient les premières victimes et les premiers témoins. Nous pouvons mentionner parmi les plus connus: The Narrative of the Adventures of Moses Roper (1838), Narrative of the life of Frederick Douglass (1845), The Narrative of William Wells Brown, A Fugitive Slave (1847), Running a Thousand Miles for Freedom (1860). Ces récits qui débutent souvent par la formule : «I was born a 
slave ", relatent la naissance de l'esclave au sein de la plantation et décrivent ses conditions de vie puis sa fuite de l'univers de la plantation jusqu'à la Terre Promise (le Nord, le Canada ou l'Angleterre) dans un mouvement physique du Sud vers le Nord (paradigme de la route vers la liberté), du rural vers l'urbain, la plantation figurant le lieu de l'immobilisme et la ville, le topos de la liberté et de la rébellion. Le narrateur qui, grâce à la Providence, jouit enfin de la liberté, devient le porte-parole des milliers de sans voix.

2 L'irruption de la voix de l'esclave inscrit sa présence sur la scène historique et universelle. La publication de ces récits, leur multiplication configure une histoire commune, un récit collectif tissé d'histoires et d'expériences singulières qui se répètent comme les motifs d'un quilt. Ces récits populaires, en dépit des omissions et des silences qui les caractérisent, créent une voix « authentique » qui vise à établir l'humanité de l'esclave. Comme le décrit l'historienne Arlette Farge, il s'agit de voix vulgaires, celles qui n'ont pas « voix au chapitre » mais qui constituent de violents signaux pour écrire l'histoire (Farge, 2009). Pourtant, si nous relisons l'histoire de l'autobiographie écrite par d'anciens esclaves entre 1830 et 1865 , nous retrouvons peu de textes écrits par des femmes. Dans la préface qu'il consacre à l'ouvrage Six Women's Slave narratives, Henry Louis Gates Jr., indique que la contribution des femmes dans le champ littéraire au XIX siècle a été minorée, en dépit du fait que la genèse de la tradition littéraire noire revienne à la poétesse Phillis Weathley (1773), que la première Africaine Américaine à publier des essais en 1841 fut Ann Plato et que Harriet E. Wilson fut la première " personne de couleur » à avoir publié un roman Our Nig (1859) (Gates, 1988, xi). À ce propos, Gates remarque que la parution de Our Nig dans le Nord rongé par le racisme d'avant-guerre, ne trouvera aucun écho au sein de l'opinion, au contraire des publications produites par d'anciens esclaves qui étaient présentées par les antiesclavagistes comme des témoignages mettant en exergue l'inhumanité de «l'Institution Particulière ».

3 Aussi, il me semble intéressant d'examiner comment la présence des voix subalternes, à la périphérie de la marge, minorées et peu étudiées ${ }^{6}$, informe le public de l'époque ainsi que le chercheur contemporain sur le désir d'inscription des narratrices dans la sphère publique, lorsqu'elles abordent la question de l'identité et de l'émancipation non plus dans le contexte du débat pro-abolitionniste mais en leur qualité de personnes libres dans une société post-esclavagiste.

4 Pour éclairer mon propos, j'ai choisi d'examiner trois récits peu connus de femmes esclaves extraits de l'ouvrage publié par Henry Louis Gates Jr. en 1988 Six Women's Slave Narratives, qui abordent la question de la présence/absence des voix de femmes dans le contexte post-esclavagiste: The Story of Mattie Jackson Written and Arranged by Dr L.S. Thompson (1866), A Slave Girl's Story Being an Autobiography of Kate Drumgoold (1898), et Memories of Chilhood's Slavery Days by Annie L.Burton (1909).

\section{Les récits d'esclaves et la question du genre}

5 Les récits d'esclaves écrits par des femmes les plus représentatifs et les plus connus, entre 1831 et 1861, sont ceux de Mary Prince ${ }^{7}$ et de Harriet Jacobs ${ }^{8}$. Winifred Morgan, dans un article consacré à la question du genre dans les récits d'esclaves du XIX siècle, pose la question de la différence de perspective et de rhétorique selon que l'auteur est un homme ou une femme (Morgan, 1994,73-94). Ainsi, Morgan démontre que dans son 
autobiographie ${ }^{9}$, Frederick Douglass se pose en tant qu'individu en quête de liberté au contraire d'Harriet Jacobs qui, comme beaucoup d'autres auteures, livre son désir d'émancipation en termes de refondation d'une communauté disséminée et brisée par le système.

6 L'une des raisons de l'absence de textes écrits par des femmes pourraient être la difficulté à se faire publier dans une société patriarcale et raciste qui accordait peu de place à l'expression féminine, comme le note Gates : « For an African American woman in the nineteenth century, publishing a book was virtually a miraculous event, as we learned from Harriet Wilson " (Crafts, 2002, lxiii). Ainsi, un certain nombre d'écrits sombrent dans l'oubli ou ne seront pas réimprimés. Grace à l'implication du Schomburg Center for Research in Black Literature, des essais, poèmes, lettres et romans écrits par des femmes après la guerre de Sécession vont surgir du silence. Près de trente ouvrages seront édités en 1987-88 (Gates, 1988, xvii). En 2001, Henry Louis Gates découvre, lors d'une vente aux enchères, le manuscrit original de l'autobiographie de Hannah Crafts écrit en 1850 qu'il publiera sous le titre de The Bondwoman's Narrative, démontrant qu'au $\mathrm{XIX}^{\mathrm{e}}$ siècle, les femmes noires, malgré leur asservissement, ont exprimé leur désir d'émancipation par le biais de l'écriture.

7 Dans le contexte de la Reconstruction, les récits d'esclaves ne s'attardent plus sur les horreurs de l'esclavage mais exigent que des efforts soient faits pour offrir plus d'opportunités d'intégration des Noirs dans la société américaine. Dans la période comprise entre la fin de la guerre de Sécession et le début $\mathrm{du} \mathrm{xx}^{\mathrm{e}}$ siècle, près de soixante-dix-sept récits seront publiés (Sekora, 1987, 483), le plus célèbre étant l'ouvrage de Booker T.Washington Up from Slavery qui parait en 1901 et dont le titre reflète la thématique du progrès de l'éducation des Noirs et de leur timide introduction dans la sphère économique. Les récits d'anciens esclaves relatant la conquête de leur liberté seront encore publiés mais l'enjeu n'est plus le même, l'esclavage ayant été aboli. Ces récits revêtent alors un caractère plutôt anthropologique. Ainsi, en tant que genre, on remarque que le récit d'esclave s'adapte aux transformations socio-politiques qui s'opèrent dans la société. Il est le reflet des ambigüités et des tensions qui sont à l'œuvre dans la société contemporaine. Dès lors, les descriptions de l'esclavage deviennent moins systématiques et constituent des preuves " historiques ». Les titres illustrent ce changement de stratégie et de rhétorique de l'ancien esclave dans une société libérée de l'esclavage: Behind the scenes: Thirty years a Slave and Four Years in the White House (Keckley, 1868), Sunshine and Shadow of Slave Life, Reminiscences Told to William Ferguson Goldie by Isaac Williams (Williams,1885), From Plantation to Congress (Langston, 1884).

8 Si nous considérons les trois représentations/ouvertures des récits de notre étude, nous observons que celle de Mattie Jackson ${ }^{10}$ correspond aux normes établies par la convention des récits d'esclaves avant l'abolition. En effet, les récits devaient obéir à certains codes. Comme le souligne Williams L. Andrews, "the most reliable slave narrative would be one that seemed purely mimetic, in which the self is on the periphery instead of the center of attention, looking outside not within, transcribing rather than interpreting a set of objective facts» (Andrews, 1986, 4). L'important paratexte renseignait sur les circonstances de l'écriture dudit récit, sa véracité : la mention « a true story » devant être apposée sur la couverture, la référence à celui qui avait retranscrit le récit dicté, le nom et l'adresse de l'imprimeur. Toutes ces informations devaient être vérifiables et conforter l'authenticité du récit. Ce qui pose la 
question de l'autorité du texte. Car de manière assez paradoxale, comme le souligne John Sekora, ce n'est pas la voix de l'esclave qui se déploie dans ces récits mais celle démultipliée de l'homme blanc : l'abolitionniste, l'imprimeur, le référent, tous ceux qui littéralement encadrent le texte et emprisonnent la voix.

Les deux autres présentations, celle de Kate Drumgoold et d'Annie L. Burton sont brèves et comme dépouillées de l'étau de l'esclavage. Ce sont des femmes-écrivains qui se souviennent; seules les références directes à l'esclavage (A Slave Girl's Story et Memories of Childhood's Slavery Days) ${ }^{11}$ éclairent le propos du texte. En effet ces deux femmes sont nées « dans » l'esclavage mais ont été libérées pendant leur enfance par les soldats de l'Union. Aucune n'a subi les brutalités de l'esclavage et chacune rend hommage aux autres femmes qui les ont entourées. Ainsi qu'elles le mentionnent en titre il s'agit de récits d'enfance. Toutefois, les trois narratrices ont en commun de relater leur volonté d'établir leur identité à part entière. Chacune développe des stratégies d'intégration différentes et, ce faisant, fait entendre une voix singulière.

Ainsi que nous l'avions mentionné précédemment, le récit de Mattie Jackson, The Story of Mattie J. Jackson, Written and Arranged by Dr. L. S. Thompson présente certaines caractéristiques propres aux récits d'esclaves $d u x^{e}{ }^{e}$ siècle $:$ la couverture, qui répond aux conventions du genre, et une préface écrite par celle qui retranscrit les mémoires et qui indique que l'objectif de ce récit est de gagner la sympathie des amis de la cause des Noirs. L'auteure remercie tous ses bienfaiteurs qui vont lui permettre, à travers l'achat de son livre, d'accéder à l'éducation. Pourtant, deux détails suggèrent un déplacement épistémologique : la référence à l'éducation comme objectif et le fait que celle qui endosse l'écriture du récit soit sa propre belle-mère, femme noire lettrée, médecin. La mention de son titre de docteur est par ailleurs synonyme du progrès social et de l'avènement timide de la bourgeoisie noire dans la période postesclavagiste. Si on se réfère au récit de Mary Prince publié en 1831, celle qui lui prêtait sa plume était une femme blanche, poétesse et amie de l'éditeur Thomas Pringle, qui restait dans l'anonymat. Le dévoilement de la transmission de l'identité (bellemère / belle-fille) illustre non seulement un changement des enjeux sociétaux mais aussi de rhétorique. Mary Prince, en 1831, prenait la parole en revendiquant son droit à la liberté. En 1866, Mattie Jackson ne craint plus de libérer sa parole. Pourtant, comme nous le verrons ultérieurement, son récit constitue un exemple de transition, d'entredeux.

Mattie Jackson naît en 1846 à Bremen, dans l'état du Missouri, de parents esclaves et décrit, chapitre après chapitre, les conditions de vie atroces de ses parents, le marronnage du père, le démembrement de la famille, les tentatives de fuite de sa mère, les conditions de vie à l'approche de la guerre, la cruauté de ses différents maîtres jusqu'à sa fuite en 1863, à l'âge de 17 ans.

12 Née en Virginie de parents esclaves en 1858 ou 1859, Kate Drumgoold est âgée de quatre ou cinq ans lorsque le président Lincoln proclame le décret d'émancipation en 1863. Elle passe peu de temps dans une plantation à Petersburg dans l'état de Virginie avec sa mère, qui est vendue et forcée de vivre en Géorgie loin de ses dix enfants disséminés dans des plantations voisines. Prise en charge par l'ancienne propriétaire de sa mère, Mrs Bettie House, Kate la retrouvera à l'issue de la guerre, ira s'installer avec elle à Brooklyn puis travaillera comme domestique. Puis vers l'âge de dix-neuf ans, elle se rend à Washington afin d'étudier au Wayland Seminary pendant quatre ans. L'autobiographie qu'elle rédige en 1898 retrace sa brève expérience d'esclave, son 
émancipation de sa position de jeune fille subalterne à celle de femme professeur et nous renseigne sur la complexité des représentations sociales de la femme noire libre au sein d'une société ségréguée ${ }^{12}$. La structure du récit de Kate Drumgoold n'obéit pas à la trame linéaire conventionnelle et rigide des textes d'esclaves. Il est entrecoupé de commentaires critiques et distanciés figurant ainsi qu'elle s'est affranchie du temps de l'esclavage et qu'elle détient désormais l'autorité de son texte et sa mémoire.

13 Annie L. Burton, qui a vécu huit années en esclavage, a peu d'expérience de la servitude puisque son enfance se déroule pendant la Guerre Civile. Née en 1858 à Clayton, en Alabama, d'une mère fugitive et d'un père blanc, Annie L. Burton illustre l'exemple de la femme noire libre industrieuse. Pour assurer son autonomie financière, elle travaillera comme domestique puis s'établira comme femme chef d'entreprise en ouvrant tour à tour un restaurant à Jacksonville (MCSD, 28), une blanchisserie à Newport (MCSD, 28) tout en prenant en charge le neveu qu'elle a recueilli après la mort de sa sœur. Elle s'établit à Boston en 1879 et se marie en 1888. Memories of Childhood's Slavery Days publié en 1909, inclut des poèmes et des hymnes qu'elle a rédigés ainsi qu'une brève biographie d'Abraham Lincoln. L'intitulé du premier chapitre de son récit peut sembler paradoxal : Recollections of a Happy Life tant les termes happy / slavery sont antinomiques. Pourtant, le trope d'une enfance insouciante pendant la guerre est commun à d'autres points de vue, tel celui de Kate Drumgoold ou encore de Lucy A. Delaney ${ }^{13}$, qui raconte : « slavery had no horror then for me, as I played about the place, with the same joyful freedom as the little white children » (13). John Blassingame, dans son ouvrage The Slave Community: Plantation Life in the Antebellum South, note que la perception de l'esclavage chez l'enfant était rendue complexe par ses relations au sein de sa famille biologique en opposition au schéma paternaliste de la famille esclave dominée par le maître (Blassingame, 1979, 181, 183-87). Au cours de sa prime enfance, l'asservissement n'était pas perçu en tant que tel par le jeune Noir qui n'avait pas conscience de son statut d'esclave. Jennifer Fleischner ajoute qu'il existe dans le souvenir de l'esclave une période " avant » qui se résume en une structure chiasmique : "liberté», esclavage, liberté, commune à beaucoup de récits d'esclaves dans les chapitres consacrés à l'enfance (Fleischner, 1996, 139). Toutefois, le récit d'Annie L. Burton est émaillé d'épisodes relatant les violents conflits qui opposent les maitres aux esclaves, les tortures infligées à des esclaves affamés-voleurs de poules, de femmes vendues parce que stériles. Ces épisodes traversent une enfance qu'elle décrit volontiers comme insouciante "happy, carefree chilhood days on the plantation " (MCSD, 3).

14 La lecture de ces voix subalternes nous livre des aspects oblitérés ou ignorés par les récits écrits par des hommes. Le lieu d'énonciation, bien qu'étant structuré de telle manière que la possibilité d'atteindre le pouvoir soit obstruée, reste un type de représentation pour celles qui ont pu échapper au silence imposé à la fois par le pouvoir colonial et par le cadre conventionnel du texte dominé par les hommes. Les expériences relatées par les hommes, dans une société patriarcale où les relations étaient hiérarchisées et segmentées, ne pouvaient que refléter un point de vue essentiellement masculin. Deborah Gray White, dans un essai intitulé Ar'n't I a Woman ?, détaille les tâches quotidiennes auxquelles les femmes étaient affectées ${ }^{14}$. Au sein de la plantation, les femmes développaient, autour des tâches différentes de celles des hommes, un réseau de coopération et d'interdépendance nécessaire à leur survie. De 
même, Betty Wood rappelle que la répartition du travail s'effectuait en fonction du sexe :

Certain skills and functions were effectively limited to men; plantation records do not reveal any women who worked as drivers, coopers, carpenters, brickmakers, and so on. Some, but by no means all, household jobs were reserved for women. On large estates upwards of 10 per cent of women were employed as cooks, maids, washerwomen, nurses, midwives, and seamstresses (Wood, 1987, 607).

Ainsi les femmes esclaves ont pu, au sein de ces fonctions séparées, développer une entraide, une solidarité féminines et transmettre un savoir-faire. Dans la plantation, les femmes esclaves évoluent généralement dans un univers féminin dans lequel s'établissent des relations d'interdépendance et de coopération (accouchement, soins etc.) Lorsqu'elles travaillent dans un même espace, les fonctions domestiques (cuisinière, matrone, accoucheuse) se transmettent de mère en fille. Les maîtres perçoivent ainsi la famille esclave composée essentiellement de la mère et de ses enfants. Le mariage entre esclaves n'amenant ni sécurité ni confort à la femme esclave, l'homme ne peut faire valoir son autorité sur son épouse. L'absence de ce type de relations a sans doute contribué à développer le sentiment d'indépendance dans le comportement quotidien des femmes.

Parce que leurs expériences de résistance à la servitude forcée sont singulières, leurs récits diffèrent des récits masculins. Winifred Morgan propose l'analyse suivante :

Black men and women, however, faced different stereotypes. Black men combated the stereotype that they were «boys " while black women contested the idea that they were either helpless victims or whores. For a male fugitive, public discourse served to claim his place among men ; for a female her relationships as a daughter, sister, wife, mother, and friend demonstrated her womanliness and her shared roles with women readers (Morgan, 1994, 76).

En outre, souligne-t-elle, les récits masculins reflètent les valeurs de la culture dominante : les femmes noires du texte de Douglass, subordonnées aux hommes, sont des exemples de victimisation; on pense au portrait que Douglass dresse de sa propre tante : « before he (my master) commenced whipping Aunt Hester, he took her into the kitchen, and stripped her from neck to waist, leaving her neck, shoulders, and back, entirely naked. He then told her to cross her hands, calling her at the same time a d----$\mathrm{d}$ b---h » (Douglass, 259). Quant à Moses Roper ${ }^{15}$, il indique dans son récit : « it will be observed, that most of the cases here cited are those in respect to males. Many instances, however, in respect to females might be mentioned but are too disgusting to appear in this narrative » (26).

En effet la représentation de la femme esclave est celle de la victime peu respectée. Présentée en offrande, mise aux enchères, anonyme, dénudée, solitaire, elle semble attendre, impavide, de devenir la chose d'un maître dépravé. Plus que l'homme, la femme esclave est associée à l'idée de dépossession de son statut d'être humain. Plus que l'homme, elle subit le processus de désontologisme propre à l'esclave, tel qu'Anny Dominique Curtius le définit dans son essai Désontologisme et réontologisme des esclaves et des marrons :

Le désontologisme, c'est la destruction de tous les paramètres socioculturels africains en fonction desquels les esclaves se reconnaissent avant la capture. En tant que processus de dépossession de l'esclave de toute son historicité, le désontologisme doit être vu comme la reconstitution d'un «être », non pas en tant qu'être humain, mais en tant qu'« être » réifié esclave (Curtius, 2000, 104). 

Deborah Gray White par exemple, affirme que la " mammy » déploie la stratégie de la préservation plus que celle de la loyauté : « Mammy knew that by becoming a friend, a confidante and indispensable servant to the Whites, she and her family might gain some immunity against sale and abuse » (Gray, 1985, 55). Cette thèse est développée par Genovese également: "Her tragedy lay, not in her abandonment of her own people, but in her inability to offer her individual power and beauty to black people on terms they could accept without sliding further into a system of paternalistic dependency " (Genovese, 1972, 361). La " mammy » correspond à l'idéal féminin victorien : vertueuse, pieuse, tendre, compréhensive, elle est plus maternelle que charnelle, telle une icône du culte de la domesticité. Selon le schéma patriarcal du XIX ${ }^{\mathrm{e}}$ siècle, elle représente la femme / esclave idéale. On lui dénie toute identité sexuelle avec l'âge (la mammy étant, par définition, vieille). Elle est aussi la pièce centrale de cette société organisée autour de l'esclavage, la gardienne du foyer, le pilier de l'institution. Cette mythification procède d'une triple oppression : elle est soumise au maître, à la maîtresse et à leurs enfants. Elle est à la fois invisible et impuissante. L'historienne Kathleen Wilson indique dans son article «Bristish Women and Empire » que, dans ce contexte, la loi infériorise les femmes noires : " law and custom worked to put white women on a pedestal that emphasized the cultural distinctions of "race" as it endowed planter society with respectability » (Wilson, 2005, 274).

22 Aussi, obligée de porter le masque obséquieux de la soumission, elle transforme son invisibilité en pouvoir. Un épisode extrait du récit de Mattie Jackson en est la parfaite illustration: ne pouvant s'opposer physiquement au fouet, sa mère le fera cuire et consommer par ses enfants : "They had a cowhide which they use to inflict on a slave girl they previously owned, nearly every night. [...] But my mother was a cook and was in the habit of roasting meats and toasting bread. As they stinted us for food my mother roasted the "cowhide" (TSMJ, 10). Il faut interpréter ce geste subversif et dérisoire comme une manière de protéger ses enfants contre le fouet et la faim mais aussi comme la mise en œuvre de la stratégie du détour. En faisant ingérer par ses enfants l'instrument de torture, elle les enjoint à «cannibaliser » l'esclavage. En transfigurant l'objet de la douleur en objet de satisfaction, cette femme y inscrit sa silencieuse résistance. La représentation de la résilience de la mère me semble une 
réponse au portrait de la femme victimisée et impuissante que Mary Prince dessine dans son récit et s'inscrit comme une rupture mimétique: "but mothers could only weep and mourn over their children, they could not save them from cruel masters, from the whip, the rope, and the cow-skin » (THMP, 197).

\section{Fille, sœur, femme, mère, amie}

Contrairement aux anciens esclaves qui pouvaient lire leurs histoires en public, les femmes étaient exclues de la sphère publique. Aussi vont-elles insister, à travers leurs récits, sur le fait qu'elles sont des filles, des sœurs, des mères, des épouses, des amies, pour s'allier la confiance du lectorat féminin blanc. Mattie Jackson annonce dans la préface :

The object in publishing this book is to gain sympathy from the earnest friends of those who have been bound down by a dominant race in circumstances over which they had no control [...]. Thus I ask you to buy my little book to aid me in obtaining an education, that I may be enabled to do some good in behalf of the elevation of my emancipated brothers and sisters (TSMJ, 3).

Pour textualiser leur propre humanité, à la différence de Mary Prince et de Harriet Jacobs qui placent le corps au centre du récit ${ }^{17}$, elles ne présentent jamais le viol ou la séduction comme des instances majeures de leur vie, elles n'exposent pas leur corps souillé mais tentent de célébrer leur part de résistance et de rébellion à l'intérieur du système. Ainsi lorsque Kate Drumgoold et Annie L. Burton relatent la douloureuse séparation d'avec leurs mères, elles les présentent comme des personnes attachées aux principes de la dignité humaine et de la liberté. La mère d'Annie, furieuse et humiliée d'avoir été fouettée par sa maîtresse, déserte la plantation pendant trois ans :

My mother was the cook in my mistress's household. One morning when master had gone to Eufaula, my mother and my mistress got into an argument, the consequence of which my mother was whipped, for the first time in her life. Whereupon, my mother refused to do any more work and ran away from the plantation. For three years we did not see her again (MCSD, 8).

À la fin de la guerre, la mère vient réclamer ses enfants et devant le refus de ses maîtres, les menace de revenir avec les soldats de l'Union « to find out if it were really true that all the negroes had been made free " (MCSD, 12). Commentant cet incident, Miya Hunter-Willis rappelle le désarroi des planteurs confrontés à la réalité de la fin d'une époque :

The presence of the Union Army threatened the slavery regime by interrupting the daily management of many Southern plantations and small estates alike. Many circumstances caused planters to worry about their property, both chattel and land. Inflicting harsher punishments to deter fugitive slaves, threatening to sell incorrigible slaves, and increasing workloads to discourage slaves from being nosy became a part of wartime life. With respect to the impact of the Civil War, Emmy Werner, a child psychologist, suggests that the lives of the slave children in the South did not change much. The presence of troops made for lively discussion amongst adults, but children were relatively unaffected unless they were sent to fugitive shelters. However, Werner states that because Missouri was part of the Union, their slaves were not considered in emancipation discussions. Promises of emancipation were guaranteed to slaves in the Confederacy (Hunter-Willis, 2008, 33). Jackson, Kate Drumgoold et Annie L.Burton mettent en exergue le rôle fondamental de 
leur mère, ses efforts pour conserver l'unité de la famille, sa lumineuse vaillance et la quête de la liberté. La mère est ainsi décrite comme une impossible fugitive, non par manque de courage mais à cause de son statut de mère. Dans l'étude consacrée au marronnage, John Hope Franklin et Loren Schweninger indiquent que la maternité est souvent considérée comme un frein au marronnage et qu'entre 1838 et $1860,81 \%$ des fugitifs étaient des hommes (Franklin et Schweninger, 1999, 210).Ils précisent que la période pendant laquelle se pratiquait la fuite se situait généralement entre l'âge de seize et trente-cinq ans. Une femme pendant ladite période était susceptible d'être enceinte ou d'allaiter. Lorsqu'elle tentait de fuir avec un groupe de fugitifs, elle était une charge pour les autres si elle était accompagnée de ses enfants :

Young slave women were less likely to run away because they had often begun to raise families by their late teens and early twenties. With youngsters to care for, it became difficult to contemplate either leaving them behind or taking them in an escape attempt. Lying out in the woods or fleeing to more distant points would only mean suffering, danger, and hardship for their children. As several historians have pointed out, although slave women desired freedom as much as slave men and were often as assertive and aggressive on the plantation as male slaves, the task of uprooting and carrying children in flight "was onerous, time-consuming, and exhaustive ». As a result, a smaller proportion than among men decided to run away (Franklin et Schweninger, 1999, 212).

Mattie Jackson raconte comment sa mère sacrifie sa soif de liberté pour préserver la cohésion de sa famille. Elle décide d'aider son mari à fuir espérant qu'un jour elle pourra, à son tour, matérialiser cette entreprise :

She concluded to assist him to make his escape from bondage. Though the parting was painful, it afforded her solace in the contemplation of her husband becoming a free man, and cherishing a hope that her little family, through the aid of some angel of mercy, might be enabled to make their escape also, and meet to part no more on earth (TSMJ, 8).

Deux années plus tard, la mère de Mattie décide de fuir avec ses deux filles mais épuisées, affamées, elles sont rattrapées au bout de trois jours, emprisonnées et vendues à un autre maître :

Two years after my father's departure, my mother, with her two children, my sister and myself, attempted to make her escape. After traveling two days we reached Illinois. We slept in the woods at night. I believe my mother had food to supply us but fasted herself [...] we were taken back to St. Louis and committed to prison (TMSJ, 9-10).

Comme dérivatif, métaphore de l'impossibilité de fuir, de maronner, certaines choisissent l'absentéisme et désertent la plantation pendant quelques jours ou quelques mois puis reviennent en servitude. C'est la pratique qu'adopte la mère d'Annie L. Burton qui, outragée d'avoir subi le fouet, abandonne la plantation et ses enfants pendant trois ans (MCSD, 8). Fuir c'est comme rompre avec la matrice, opérer l'avortement du système et ces mères-là s'y refusent loin de la fulgurance de femmes telles Harriet Tubman ${ }^{18}$ ou Sojourner Truth ${ }^{19}$.

Le thème majeur qui relie ces récits est la présentation de la mère comme l'épitomé de la résistance. De victime passive qu'elle figure dans les récits masculins, elle devient une héroïne silencieuse et déterminée. Parce que les femmes sont au centre du récit, l'image que nous proposent ces narratrices est plus complexe. Ces auteures développent ainsi des portraits plus élaborés des personnages féminins et s'attachent davantage à l'esprit de résistance qu'au corps victimisé : quand elles décrivent leurs 
expériences malheureuses c'est pour en puiser force et ténacité. Mattie confesse, par exemple, qu'elle a dû dérober vingt-cinq dollars à son maître la veille de sa fuite afin de «faire face à des frais » (TSMJ, 38). Il peut paraitre surprenant que pareil « péché » soit énoncé sans l'ombre d'un repentir dans un récit émaillé de références religieuses. C'est, sans doute, sa propre manière de résister et de dire que la liberté prime. Le récit de Mattie constitue, avant tout, un appel à la justice. Elle décide de s'octroyer son dû car, semble-t-elle dire, une femme noire n'est en rien redevable de l'esclavage. Les différents épisodes ou incidents qui rythment son texte démontrent comment cette voix subalterne exige que son auditoire reconnaisse son récit à la fois comme témoignage des horreurs de l'esclavage et comme la prise de conscience de son identité de femme. En 1863, la famille est disséminée, sa sœur, sa mère et son jeune frère ayant été vendus à des maitres différents. Dans l'immense demeure du Captain Ephraim Frisbee, elle s'épuise à la tâche et rapporte: «I was then seventeen years of age. My health has been impaired from that time to the present. [...] I am infirm and burdened with the influence of slavery, whose impress will ever remain on my mind and body " (TSMJ, 26). Quelques années plus tard, à l'issue de la guerre, elle s'installe à Indianapolis et commente son émancipation en ces termes :

I had always been under the yoke of oppression, compelled to submit to its laws, and not allowed to advance a rod from the house, or even out of call, without a severe punishment. Now this constant fear and restless yearning was over. It appeared as though I had emerged into a new world, or had never lived in the old one before (TSMJ, 28).

Le récit de Kate Drumgoold est intéressant en ce qu'il aborde une expérience particulière qui a été rarement relatée dans les textes d'esclaves : elle est à la fois la fille de l'esclave noire qui l'a enfantée et de la maîtresse blanche qui l'a élevée : «I was three years old when I was leaving my own dear mother's home to go to my new mother's home, or should I say to my white mother's home, to live with her » (ASGS, 29). Sa mère est vendue au début de la guerre par un maître désireux d'échapper à la conscription (le produit de cette vente lui permettra d'envoyer un autre homme à sa place). Un couple de Blancs, Mr et Mrs House les prend en charge, sa sœur et elle, et les traite comme ses propres enfants. L'affection que Kate porte à cette mère de substitution semble indéfectible comme en témoignent ces nombreuses allusions : «my dear white mother » (ASGS, 11), « my dearly beloved white mother » (ASGS, 13). Lorsqu'elle parle de sa génitrice noire, Kate emploie les termes de « dear mother » $(A S G S, 9,18)$ ou de « slave mother » $(A S G S, 12)$ car elle ne la célèbre pas moins en la décrivant comme une femme consciente de sa dignité de personne qui cherche par tous les moyens à rassembler sa nombreuse progéniture (dix filles et un fils) disséminée sur plusieurs plantations : « My mother was one that the master could not do anything to make her feel like a slave and she would battle with them to the last that she would not recognize them as her lord and master and she was right » (ASGS, 29).

32 Cet exemple illustre le fait que les représentations maternelles de ce type ne sont pas figées dans un schéma manichéen: maîtresse blanche malveillante/mère noire bienveillante, représentations que nous retrouvons dans le récit de Mattie Jackson. Nous pouvons ajouter qu'il s'agit ici d'une socialisation à l'inverse de celle que pratiquait la «mammy» dans ses rapports avec les enfants du maître qui lui étaient confiés, comme le précise Deborah Gray White :

White women also played a role in slave child care, and while a legend has been

built around the black nurses who helped raise Southern white children, the role 
that white women played in raising slave children has largely been ignored [...]. Tradition holds that the black Mammy socialized her white charges, but a much overlooked aspect of the Southern mistress's activities was the role she played in the socialization of black children (Gray White, 1985, 53).

Jennifer Fleischner, qui aborde cette double relation à la mère sous l'angle psychanalytique, estime que le système esclavagiste brouille les rapports du modèle mère / fille car il sépare la mère de la fille et force la fille à se trouver une mère de substitution qui lui apporterait amour et réconfort. Ainsi le modèle paradoxal qui résulte de cette figure maternelle brisée d'un point de vue racial, contribue à élaborer un questionnement complexe à l'identification et à l'identité :

This turning toward the "white mother", which involves a turning against her unChristian and culturally denigrated black mother, amounts to a violation of her own primary identity, her original bond to and identification with her own mother. Indeed, to turn toward the black mother is a turning against what she perceives to be the potential of motherhood, as well as the cultural ideal embodied in the white woman; but to turn toward the white mother is to turn against herself. Hence a kind of self-fragmentation is her fate (Fleischner, 1996, 48).

On pourrait analyser la structure non-chronologique du récit de Kate Drumgoold comme une mise en œuvre de cette tension entre ces deux figures contrastées de la mère, et comme une inscription de sa capacité individuelle à réorganiser sa mémoire. Cette surreprésentation de la mère dessine en creux l'absence du père et semble conforter le mythe controversé de la famille esclave défaillante et désorganisée ${ }^{20}$.

Kate Drumgoold raconte comment, sa mère ayant été vendue sur une autre plantation, son père épouse une autre femme : «my dear mother had a dear husband that she was sold from also, and he, not knowing that he should see my mother any more, as the times were then, he waited for a while and then he found him another wife » (ASGS, 9). Quant au père de Mattie, il fuit la plantation : « on this Saturday evening he arrived and gave us all his farewell kiss, and hurried away » (TSMJ, 9). Sa mère se marie de nouveau et se trouve une nouvelle fois abandonnée par son second mari qui fuit au Canada. Annie L. Burton ne fait qu'une brève et sèche référence à son père : «my mistress told me that my father was a planter who owned a plantation about two miles from ours. He was a white man, born in Liverpool, England » $(M C S D, 7)$ et, plus loin dans le texte, elle souligne en creux son statut de fille bâtarde: "I never knew my father, who was a white man » (MCSD, 37). Ces pères fantômes sont des produits du système esclavagiste qui forcent certains esclaves à fuir l'enfermement de la plantation (le père de Mattie, celui de Kate) ou à reproduire la figure de domination masculine (le père d'Annie). Ainsi la matrilinéarité s'opère à travers le lien de l'écrit. C'est la mère qui enfante le récit de Mattie qui, à son tour, par le biais d'une mise en abyme, raconte les efforts conjugués des deux narratrices (sa belle-mère et elle-même) pour "accoucher » du texte :

I had previously told my step-mother my story and how often my own mother had wished she could have it published. I did not imagine she could have time to write and arrange it, but she immediately proposed writing and publishing the entire story, by the sale of which I might obtain the aid towards completing my studies (TSMJ, 36).

Au-delà de la parole libérée, mère et belle-mère incitent Mattie à revendiquer une double émancipation, noire et féminine à la fois, par le truchement de l'éducation. Elle ne raconte pas pour témoigner mais plus encore pour gagner son statut de femme libre. L. Burton rapporte qu'en 1866, alors qu'elle vivait dans une cabane avec sa mère et ses 
quatre frères et sœurs, Mis' Mary, une femme riche à la recherche d'une jeune servante pour sa fille, l'emploie et décide toutefois de l'envoyer à l'école du dimanche puis de lui enseigner chaque jour la lecture et l'écriture : «my new mistress sent me to Sunday school every Sunday morning, and I soon got so that I could read 》 (MCSD, 13).

Nous réalisons à travers ces exemples que le corps, surface d'impression qui porte les marques de l'esclavage est ici oblitéré, dé-marqué, car la marque importante, celle qui doit faire événement est celle qui s'inscrit sur la page. L'encre remplace le sang.

Ce qui anime donc toutes ces femmes esclaves c'est la nécessité de sortir de la nuit de l'esclavage, de transmettre une nouvelle posture dans cette société post-esclavagiste et d'y marquer leur empreinte. À la fin de son récit, Mattie, après avoir non sans ironie remercié son maître qui lui a permis l'accès à l'instruction, promet de publier les détails de son histoire de sa propre plume marquant ainsi la conquête de son identité. Kate Drumgoold s'installe à New York avec sa mère et se consacre à la promotion de sa race en devenant enseignante. Annie devient une femme d'entreprise à Boston et, comme les autres femmes qui lui ont servi de modèle, prend en charge l'éducation de son neveu. Cette conquête de l'espace figure une ascension sociale.

En écrivant leurs autobiographies, ces femmes ont mis en œuvre leur vision d'un monde auquel elles ont participé et ont donné à voir les représentations qui correspondaient aux expériences qu'elles ont traversées. Elles disent qu'elles aussi ont mêlé leurs voix à la revendication de la liberté de leur race et que l'affirmation d'un rôle propre aux femmes est indispensable. Elles exposent dans la sphère publique la résistance entamée par leurs mères dans la sphère privée de la plantation. À travers ces portraits de femmes et, singulièrement, ceux de leurs mères, ordinaires et admirables, elles demandent que leur présence soit désormais inscrite en toute lumière dans le combat pour l'émancipation de la race noire aux États-Unis. Elles se déterminent comme des défricheuses de ce nouvel espace de liberté qu'elles doivent conquérir par le biais de l'écriture. Parce qu'elles ont connu l'entre-deux de l'asservissement et de la liberté, ayant vécu l'esclavage au cours de leur enfance, leur projet d'écriture se révèle plus complexe. Kate Drumgoold, Mattie Jackson et Annie L. Burton sont des actrices historiques et littéraires de l'histoire de l'esclavage aux États-Unis. Leurs récits sont remarquables en ce qu'ils permettent la compréhension d'histoires ignorées, inaudibles. L'acceptation de ces voix nous invite à reconnaître leur présence dans la communauté humaine.

\section{BIBLIOGRAPHIE}

ANDREWS, William L., To Tell a Free Story: The First Century of Afro-American Autobiography 1760-1865, Urbana, University of Illinois Press,1986.

BERLIN, Ira, Many Thousands Gone: The First Two Centuries of Slavery in North America, Cambridge (MA) et Londres, Belknap Press of Harvard University Press, 1998. 
BLASSINGAME, John, The Slave Community: Plantation Life in the Antebellum South, New York et Oxford, Oxford University Press,1979.

CRAFTS, Hannah, The Bondwoman's Narrative, A Novel, Henry Louis Gates Jr., dir., New York, Warner Books, 2002.

CURTIUS, Anny, « Désontologisme et réontologisme des esclaves et des marrons » dans Bernabé, Jean et al, dir., Des îles créoles aux sociétés plurielles, Cayenne, Ibis rouge éditions, 2000.

FARGE, Arlette, Essai pour une histoire des voix au dix-huitième siècle, Paris, Bayard éditions, 2009.

FLEISCHNER, Jennifer, Mastering Slavery. Memory, Family, and Identity in Women's Slave Narratives, New York, New York University Press, 1996.

FOSTER, Frances Smith, Witnessing Slavery, the Development of Ante-Bellum Slave Narratives, Madison, University of Wisconsin Press, 1979.

FRANKLIN, John Hope et Loren SCHWENINGER, Runaway Slaves. Rebels on the Plantation, New York, Oxford University Press, 1999.

FRAZIER, Franklin E.,The Negro family in the U.S, Chicago, Chicago University Press, 1939.

FRUND, Arlette, Écritures d'esclaves, Phillis Wheatley \& Olaudah Equiano, figures pionnières de la diaspora africaine américaine, Paris, Michel Houdiard Editeur, 2007.

GATES, Henry Louis Jr., Figures in Black: words, signs, and the « racial » self, New York, Oxford University Press, 1987.

---, ed., The Classic Slave Narratives, New York, Mentor Penguin Books, 1987.

---, ed.,Six women's slave narratives, New York, Oxford University Press, 1988.

GRAY WHITE, Deborah, Ar'n't I a woman? Female slaves in the plantation South, New York, W.W. Norton \& Company, 1985.

GENOVESE, Eugene, D.,Roll, Jordan, Roll, The World the Slaves Made,New York, Ramdon House, 1972.

HUNTER-WILLIS, Miya, Writing the Wrongs. A Comparison of two Slave Narratives, Marshall University, 2008, http://muwww-new.marshall.edu/etd/masters/hunter-willis-miya-2008ma.pdf (page consultée le 10 avril 2013).

JONES, Gayl, African American Literature, Cambridge, Havard University Press, 1991

MORGAN, Winifred, « Gender-related difference in the slave narratives of Harriet Jacobs and Frederick Douglass », American Studies, 35, 1994, 73-94.

SEKORA, John, «Black Message/White Envelope : Genre, Authenticity and Authority in the Antebellum Slave Narrative », Callaloo, 32, Summer 1987, 482-515.

SEKORA, John et Darwin T. TURNER, The Art of the Slave Narrative : Original Essays in Criticism and Theory, Macomb (ILL), Western Illinois University, 1982.

WERNER, Emmy, Reluctant Witness : Children's Voices from the Civil War, Boulder, Westview Press, 1988.

WILSON, Kathleen, « British Women and Empire », Women's History : Britain, 1700-1850: An Introduction, Hannah Barker et Elaine Chalus, dir., Londres et New York, Routledge, 2005.

WOOD, Betty, « Some Aspects of Female Resistance to Chattel Slavery in Low Country Georgia, 1763-1815, » The Historical Journal, vol. 30, n³, Sep.1987, 603-22. 


\section{NOTES}

1. Jusqu'en 1807, date de l'abolition de la traite transatlantique en Angleterre.

2. Historien de renom, Henry Louis Gates Jr., auteur de plusieurs ouvrages sur les récits d'esclavages a développé l'intérêt de nombreux chercheurs pour ce champ alors inexploité (Gates, 1987, x).

3. À noter que le Parlement britannique abolit l'esclavage dans toutes ses colonies en 1833 et que les anti-esclavagistes britanniques vont soutenir la lutte des Américains dans leur attaque contre l'institution de l'esclavage.

4. Il s'agit du premier recueil de poésie rédigé par une esclave, Phillis Wheatley (1753-1784) considérée comme la première poétesse africaine américaine.

5. John Sekora et D.Turner, The Art of the Slave Narrative (1982), analysent les effets rhétoriques des récits d'esclaves ; Frances Smith Foster, Witnessing Slavery (1979), explore le genre ; William L. Andrews, To tell a Free Story: the first Century of Afro-American Autobiography, 1760-1865 (1988), examine les récits en tant qu'autobiographies publiques ayant pour finalité la liberté.

6. À mentionner : un mémoire de Master d'histoire «Writing the Wrongs : A comparison of two female slave narratives », Miya Hunter-Willis, Marshall University, 2008. Le récit de Kate Drumgoold fait aussi l'objet d'une analyse dans « Enduring Memory; Kate Drumgoold and Julia A.J.Foote » (Fleischner, 1996, 133-53).

7. The History of Mary Prince, A West-Indian Slave, 1831, publié dans The Classic Slave Narratives (Gates, 1987a).

8. Incidents in the Life of a Slave Girl, Written by Herself, 1861, publié dans The Classic Slave Narratives.

9. Narrative of the Life of Frederick Douglass, An African Slave, 1845.

10. The Story of Mattie Jackson, 1866.

11. Le récit de Burton sera désormais désigné par les initiales du titre MCSD, celui de Jackson par les initiales TSMJ.

12. En dépit du $13^{\mathrm{e}}$ Amendement qui interdit toute servitude forcée, des pratiques seront instituées pour restreindre la liberté des anciens esclaves. Ni le Civil Rights Act (1866), ni le $14^{\mathrm{e}}$ Amendement (1868), ne pourront éradiquer les injustices. En 1896, l'arrêt Plessey vs Ferguson valide la ségrégation de manière institutionnelle.

13. From The Darkness Cometh the Light or Struggles for Freedom (1891) publié dans Six Women's Slave Narratives.

14. Gray White, 1985, 75, 122-24. Le titre de l'ouvrage de Gray White est emprunté au célèbre discours prononcé en 1851 par Sojourner Truth, militante noire féministe (1797-1883).

15. A Narrative of the Adventures and Escape of Moses Roper from American Slavery, 1838.

16. On peut mentionner Edward A. Pollard qui écrit dans sa défense du système esclavagiste "Aunt Debby is an aged colored female of the very highest respectability. [...] she slept in the Big House and referred to slaves of lesser social position as "de niggers" " Black Diamonds (22) cité dans Genovese, 1972, 355.

17. Harriet Jacobs raconte en détail les avances de son maître Dr Flint ; elle finira par accepter, pour se protéger, de devenir la maîtresse d'un autre Blanc : «I knew what I did, and did it with deliberate calculation » (54). Quant à Mary Prince, elle raconte l'humiliante séance du bain d'un de ses maitres en ces termes: " he had an ugly fashion of stripping himself quite naked, and ordering me then to wash him in a tub of water. This was worse to me than all the licks " (202).

18. (1820-1913) esclave fugitive qui par le biais de l'organisation de l'Underground Railroad, contribua à l'évasion de centaines d'esclaves.

19. (1797-1883) affranchie en 1828, conférencière, militante anti-esclavagiste et féministe.

20. Voir l'excellente analyse de Genovese sur la question dans son ouvrage (Genovese, 1972, 451-57). 


\section{RÉSUMÉS}

Cet article propose d'examiner comment la présence des voix de femmes esclaves, à la périphérie de la marge, minorées et peu étudiées, informe le public de l'époque ainsi que le chercheur contemporain sur le désir d'inscription des narratrices dans la sphère publique, lorsqu'elles abordent à travers leurs textes la question de l'identité et de l'émancipation, non plus dans le contexte du débat pro-abolitionniste mais en leur qualité de personnes libres dans une société post-esclavagiste.

This essay examines the presence of the half-muted and marginalized women slaves' voices which nevertheless inform the audience of the time as well as the contemporary scholar on their desire to inscribe their being within the public sphere as they question the issue of identity and emancipation through their texts. Beyond the pro-abolitionist debate context, they speak as true and free human beings living in a post-slavery society.

\section{INDEX}

Mots-clés : esclavage, récits d'esclaves, voix, identité, émancipation

Keywords : slavery, slave narratives, voice, identity, emancipation

\section{AUTEUR}

\section{DOMINIQUE AURÉLIA}

Université des Antilles et de la Guyane 